\title{
On the order of strong starlikeness and the radii of starlikeness for of some close-to-convex functions
}

\author{
Mamoru Nunokawa ${ }^{1} \cdot$ Janusz Sokół² \\ Received: 10 February 2018 / Revised: 5 July 2019 / Accepted: 5 September 2019 / \\ Published online: 13 September 2019 \\ (c) The Author(s) 2019
}

\begin{abstract}
In this paper we show several sufficient conditions for close-to-convex functions to be strongly starlike of some order. The results continue the line of study from the first author's paper on the order of strong starlikeness of strongly convex functions, (Nunokawa in Proc Japan Acad Ser A 69(7):234-237, 1993). Also it appears an small improvement of a certain classical results of $\mathrm{Ch}$. Pommerenke. As an application, we also derive estimates for the radii of star-likeness for close-to-convex functions.
\end{abstract}

Keywords Analytic functions · Convex functions · Starlike functions · Univalent functions · Strongly starlike

Mathematics Subject Classification Primary 30C45 · Secondary 30C80

\section{Introduction}

Let $\mathcal{H}$ denote the class of analytic functions in the unit disc $\mathbb{D}=\{z:|z|<1\}$ on the complex plane $\mathbb{C}$. Let $\mathcal{A}$ denote the subclass of $\mathcal{H}$ consisting of functions normalized by $f(0)=0, f^{\prime}(0)=1$. The set of all functions $f \in \mathcal{A}$ that are convex univalent in $\mathbb{D}$ we denote by $\mathcal{K}$. The set of all functions $f \in \mathcal{A}$ that are starlike univalent in $\mathbb{D}$ with respect to the origin we denote by $\mathcal{S}^{*}$. Recall that a set $E \subset \mathbb{C}$ is said to be starlike with respect to a point $w_{0} \in E$ if and only if the line segment joining $w_{0}$ to any other point $w \in E$ lies entirely in $E$. A set $E$ is said to be convex if and only if it is starlike with respect to each of its points, that is if and only if the linear segment joining any

\footnotetext{
\Janusz Sokół

jsokol@ur.edu.pl

Mamoru Nunokawa

mamoru_nuno@doctor.nifty.jp

1 University of Gunma, Hoshikuki-cho 798-8, Chuou-Ward, Chiba 260-0808, Japan

2 Faculty of Mathematics and Natural Sciences, University of Rzeszów, ul. Prof. Pigonia 1, 35-310 Rzeszów, Poland
} 
two points of $E$ lies entirely in $E$. An univalent function $f$ maps $\mathbb{D}$ onto a convex domain $E$ if and only if [11]

$$
\mathfrak{R e}\left\{1+\frac{z f^{\prime \prime}(z)}{f^{\prime}(z)}\right\}>0 \text { for all } z \in \mathbb{D} .
$$

Such a function $f$ is said to be convex in $\mathbb{D}$ (or briefly convex). In [8] Sakaguchi proved that if $f \in \mathcal{A}$ and $g \in \mathcal{S}^{*}$, then

$$
\left[\mathfrak{R e}\left\{\frac{f^{\prime}(z)}{g^{\prime}(z)}\right\}>0, \quad z \in \mathbb{D}\right] \Rightarrow\left[\mathfrak{R e}\left\{\frac{f(z)}{g(z)}\right\}>0, \quad z \in \mathbb{D}\right] .
$$

This result was also generalized, see [2] and [7]. In [6] Pommerenke established a formula for $\beta=\beta(\alpha)$ such that

$$
\left|\operatorname{Arg}\left\{\frac{f^{\prime}(z)}{g^{\prime}(z)}\right\}\right|<\frac{\alpha \pi}{2}, \quad z \in \mathbb{D} \Rightarrow\left|\operatorname{Arg}\left\{\frac{f(z)}{g(z)}\right\}\right|<\frac{\beta \pi}{2}, \quad z \in \mathbb{D},
$$

where $f(z) \in \mathcal{K}_{\alpha}$. This is a generalization of the relation of type (1.1) because is in the class $\mathcal{K}_{\alpha} \alpha, 0<\alpha \leq 1$ whenever $f(z) \in \mathcal{A}$ and there exist a function $g(z) \in \mathcal{K}$ such that

$$
\left|\operatorname{Arg}\left\{\frac{f^{\prime}(z)}{g^{\prime}(z)}\right\}\right|<\frac{\alpha \pi}{2}, \quad z \in \mathbb{D} .
$$

Here we understand that $\operatorname{Arg} w$ is a number in $(-\pi, \pi]$. It is known that if $f(z) \in \mathcal{K}_{\alpha}$, then $f(z)$ is close-to-convex and so $f(z)$ is univalent in $\mathbb{D}$. The class $f(z) \in \mathcal{K}_{\alpha}$ is called the class of strongly close-to-convex functions of order $\alpha$.

This result has found many applications. Condition (1.2) with $g(z)=f(z)$ becomes

$$
\left|\operatorname{Arg}\left\{\frac{z f^{\prime}(z)}{f(z)}\right\}\right|<\frac{\pi \alpha}{2}, \quad z \in \mathbb{D},
$$

and it says that $f(z)$ is a strongly starlike function of order $\alpha, 0<\alpha \leq 1$. The class of strongly starlike functions was introduced in $[1,10]$, we denote this class here by $\mathcal{S}^{*}(\alpha)$. One can consider functions satisfying condition (1.3) with $0<\alpha<2$ and in this case we will named such functions also strongly starlike of order $\alpha, 0<\alpha<2$. It is known that if $f(z)$ is strongly starlike of order $\alpha>1$, then $f(z)$ need not to be univalent in $\mathbb{D}$.

We say that $f(z) \in \mathcal{K}_{\beta}^{\alpha}$ whenever $f(z) \in \mathcal{A}$ and there exists a real $\alpha, 0<\alpha \leq 1$ and a function $g(z) \in \mathcal{S}^{*}(\beta) \cap \mathcal{K}, 0<\beta \leq 1$, such that

$$
\left|\operatorname{Arg}\left\{\frac{f^{\prime}(z)}{g^{\prime}(z)}\right\}\right|<\frac{\alpha \pi}{2}, \quad z \in \mathbb{D} .
$$

Lemma 1.1 Let $f(z) \in \mathcal{K}_{\beta}^{\alpha}$ with $0<2 \alpha+\beta \leq 1,0<\alpha \leq 1,0<\beta \leq 1$. Then we have 


$$
\left|\operatorname{Arg}\left\{\frac{z f^{\prime}(z)}{f(z)}\right\}\right|<\frac{\pi}{2}(\beta+2 \alpha), \quad z \in \mathbb{D},
$$

or $f(z)$ is strongly starlike function, of order $\beta+2 \alpha$.

Proof If $f(z) \in \mathcal{K}_{\beta}^{\alpha}$, then $f(z)$ is univalent in $\mathbb{D}$ and so

$$
\operatorname{Arg}\left\{\frac{z f^{\prime}(z)}{f(z)}\right\}
$$

exists for all $z \in \mathbb{D}$. It is known the following Pommerenke's result [6, Lemma 1, p. 180]: If $f(z)$ is analytic and $g(z)$ is convex in $\mathbb{D}$, then

$$
\begin{gathered}
\exists \alpha \in(0,1] \forall z \in \mathbb{D}:\left|\operatorname{Arg} \frac{f^{\prime}(z)}{g^{\prime}(z)}\right|<\frac{\alpha \pi}{2} \\
\Rightarrow \forall z_{1}, z_{2} \in \mathbb{D}:\left|\operatorname{Arg} \frac{f\left(z_{2}\right)-f\left(z_{1}\right)}{g\left(z_{2}\right)-g\left(z_{1}\right)}\right|<\frac{\alpha \pi}{2} .
\end{gathered}
$$

Applying this Lemma with $z_{1}=0$ gives

$$
\begin{aligned}
\operatorname{Arg}\left\{\frac{z f^{\prime}(z)}{f(z)}\right\} & =\operatorname{Arg}\left\{\frac{f^{\prime}(z)}{g^{\prime}(z)} \frac{z g^{\prime}(z)}{g(z)} \frac{g(z)}{f(z)}\right\} \\
& \leq \operatorname{Arg}\left\{\frac{f^{\prime}(z)}{g^{\prime}(z)}\right\}+\operatorname{Arg}\left\{\frac{z g^{\prime}(z)}{g(z)}\right\}+\operatorname{Arg}\left\{\frac{g(z)}{f(z)}\right\},
\end{aligned}
$$

it follows that

$$
\begin{aligned}
\left|\operatorname{Arg}\left\{\frac{z f^{\prime}(z)}{f(z)}\right\}\right| & \leq\left|\operatorname{Arg}\left\{\frac{f^{\prime}(z)}{g^{\prime}(z)}\right\}\right|+\left|\operatorname{Arg}\left\{\frac{z g^{\prime}(z)}{g(z)}\right\}\right|+\left|\operatorname{Arg}\left\{\frac{g(z)}{f(z)}\right\}\right| \\
& <\frac{\alpha \pi}{2}+\frac{\beta \pi}{2}+\frac{\alpha \pi}{2} \\
& =\frac{\pi}{2}(\beta+2 \alpha) .
\end{aligned}
$$

We note that a result of the form related to (1.5) was proved in [5, Theorem 2.1].

Lemma 1.2 Let $h(z)=1+\sum_{n=1}^{\infty} c_{n} z^{n}$ be in $\mathcal{H}$. If

$$
|\operatorname{Arg}\{h(z)\}|<\frac{\alpha \pi}{2}, \quad z \in \mathbb{D},
$$

for some $\alpha>0$, then

$$
|\operatorname{Arg}\{h(z)\}|<\alpha \sin ^{-1} \frac{2|z|}{1+|z|^{2}}, \quad z \in \mathbb{D},
$$


and

$$
\mathfrak{R e}\left\{h^{1 / \alpha}(z)\right\}>\frac{1-|z|}{1+|z|}, \quad z \in \mathbb{D} .
$$

Proof From the hypothesis $h(z) \neq 0$, so the function

$$
h^{1 / \alpha}(z), \quad h^{1 / \alpha}(0)=1,
$$

is in the class $\mathcal{H}$. Furthermore, we have

$$
\mathfrak{R e}\left\{h^{1 / \alpha}(z)\right\}>0, \quad z \in \mathbb{D}
$$

hence

$$
h^{1 / \alpha}(z) \prec \frac{1+z}{1-z},
$$

and $h^{1 / \alpha}(z)$ is contained in the circle with the radius and center

$$
R=\frac{2|z|}{1-|z|^{2}} \quad \text { and } \quad C=\frac{1+|z|^{2}}{1-|z|^{2}}
$$

respectively. From this, we obtain (1.7) and (1.8).

If we take $g(z)=z$ then Pommerenke's result (1.5) becomes the following corollary.

Corollary 1.3 Let $f(z)$ be in $\mathcal{A}$. If

$$
\left|\operatorname{Arg}\left\{f^{\prime}(z)\right\}\right|<\frac{\alpha \pi}{2}, \quad z \in \mathbb{D}
$$

for some $\alpha, 0<\alpha \leq 1$, then

$$
\left|\operatorname{Arg}\left\{\frac{f(z)}{z}\right\}\right|<\frac{\alpha \pi}{2}, \quad z \in \mathbb{D} .
$$

In the next Corollary we extend $\alpha$ to $1<\alpha<2$ :

Corollary 1.4 Let $f(z)$ be in $\mathcal{A}$. If

$$
\left|\operatorname{Arg}\left\{f^{\prime}(z)\right\}\right|<\frac{\alpha \pi}{2}, \quad z \in \mathbb{D},
$$

for some $\alpha, 1<\alpha \leq 2$, then

$$
\left|\operatorname{Arg}\left\{\frac{f(z)}{z}\right\}\right|<\frac{\alpha \pi}{2}, \quad z \in \mathbb{D} .
$$


Proof For arbitrary $z \in \mathbb{D}$ and from the hypothesis $1<\alpha \leq 2$, can connect the point $f^{\prime}(z)$ and $f^{\prime}(0)=1$ by a line segment as $\overline{f^{\prime}(z) f^{\prime}(0)}=\overline{f^{\prime}(z) 1}$. Applying the same method as in the proof of Pommerenke [6, p. 180], we have

$$
\frac{f(z)}{z}=\frac{f(z)-f(0)}{z-0}=\int_{0}^{1} f^{\prime}(0+t z) \mathrm{d} t
$$

and so, applying the property of integral mean, we have

$$
\left|\operatorname{Arg}\left\{\frac{f(z)}{z}\right\}\right|<\frac{\alpha \pi}{2}, \quad z \in \mathbb{D} \text {. }
$$

To prove the main results, we also need the following generalization of the Nunokawa's Lemma, [3,4].

Lemma 1.5 [5] Let $p(z)$ be analytic function in $|z|<1$ of the form

$$
p(z)=1+\sum_{n=k}^{\infty} a_{n} z^{n}, \quad a_{k} \neq 0,
$$

with $p(z) \neq 0$ in $|z|<1$. If there exists a point $z_{0},\left|z_{0}\right|<1$, such that

$$
|\operatorname{Arg}\{p(z)\}|<\frac{\pi \alpha}{2} \text { for }|z|<\left|z_{0}\right|
$$

and

$$
\left|\operatorname{Arg}\left\{p\left(z_{0}\right)\right\}\right|=\frac{\pi \alpha}{2}
$$

for some $\alpha>0$, then we have

$$
\frac{z_{0} p^{\prime}\left(z_{0}\right)}{p\left(z_{0}\right)}=i m \alpha
$$

where

$$
m \geq \frac{k}{2}\left(a+\frac{1}{a}\right) \geq k \text { when } \operatorname{Arg}\left\{p\left(z_{0}\right)\right\}=\frac{\pi \alpha}{2}
$$

and

$$
m \leq-\frac{k}{2}\left(a+\frac{1}{a}\right) \leq-k \text { when } \operatorname{Arg}\left\{p\left(z_{0}\right)\right\}=-\frac{\pi \alpha}{2}
$$

where

$$
\left\{p\left(z_{0}\right)\right\}^{1 / \alpha}= \pm i a, \text { and } a>0 .
$$

Theorem 1.6 Let $f(z)$ and $g(z)$ be in $\mathcal{A}$. Suppose that

$$
\left|\operatorname{Arg}\left\{\frac{f^{\prime}(z)}{g^{\prime}(z)}\right\}\right|<\frac{\alpha \pi}{2}+\tan ^{-1} \frac{\alpha \beta \cos \left\{\operatorname{Arg} \frac{g(z)}{z g^{\prime}(z)}\right\}}{1-\alpha \beta \sin \left\{\operatorname{Arg} \frac{g(z)}{z g^{\prime}(z)}\right\}}, \quad z \in \mathbb{D},
$$


for some $\alpha$ and $\beta$ such that $0<\alpha$ and $0<\beta<1$. Assume also

$$
\left|\frac{g(z)}{z g^{\prime}(z)}\right|>\beta, \quad z \in \mathbb{D},
$$

then

$$
\left|\operatorname{Arg}\left\{\frac{f(z)}{g(z)}\right\}\right|<\frac{\alpha \pi}{2}, \quad z \in \mathbb{D} .
$$

Proof Let us write

$$
p(z)=\frac{f(z)}{g(z)}
$$

then we have

$$
\frac{f^{\prime}(z)}{g^{\prime}(z)}=p(z)\left(1+\frac{z p^{\prime}(z)}{p(z)} \frac{g(z)}{z g^{\prime}(z)}\right)
$$

and

$$
\begin{aligned}
\operatorname{Arg}\left\{\frac{f^{\prime}(z)}{g^{\prime}(z)}\right\} & =\operatorname{Arg}\left\{p(z)\left(1+\frac{z p^{\prime}(z)}{p(z)} \frac{g(z)}{z g^{\prime}(z)}\right)\right\} \\
& =\operatorname{Arg}\{p(z)\}+\operatorname{Arg}\left\{1+\frac{z p^{\prime}(z)}{p(z)} \frac{g(z)}{z g^{\prime}(z)}\right\} .
\end{aligned}
$$

If there exists $z_{0} \in \mathbb{D}$ such that

$$
|\operatorname{Arg}\{p(z)\}|<\frac{\alpha \pi}{2}, \quad|z|<\left|z_{0}\right|
$$

and

$$
\left|\operatorname{Arg}\left\{p\left(z_{0}\right)\right\}\right|=\frac{\alpha \pi}{2},
$$

then from Lemma 1.5

$$
\frac{z p^{\prime}\left(z_{0}\right)}{p\left(z_{0}\right)}=i \alpha k
$$

for some $k \geq 1$ when $\operatorname{Arg}\left\{p\left(z_{0}\right)\right\}=\alpha \pi / 2$ or for some $k \leq-1$ when $\operatorname{Arg}\left\{p\left(z_{0}\right)\right\}=$ $-\alpha \pi / 2$. For the case $\operatorname{Arg}\left\{p\left(z_{0}\right)\right\}=\alpha \pi / 2$, from (1.12), we have

$$
\begin{aligned}
& \operatorname{Arg}\left\{1+\frac{z_{0} p^{\prime}\left(z_{0}\right)}{p\left(z_{0}\right)} \frac{g\left(z_{0}\right)}{z_{0} g^{\prime}\left(z_{0}\right)}\right\}=\operatorname{Arg}\left\{1+i \alpha k \frac{g\left(z_{0}\right)}{z_{0} g^{\prime}\left(z_{0}\right)}\right\} \\
& >\operatorname{Arg}\left[1+i \alpha \beta k\left(\cos \left\{\operatorname{Arg} \frac{g\left(z_{0}\right)}{z_{0} g^{\prime}\left(z_{0}\right)}\right\}+i \sin \left\{\operatorname{Arg} \frac{g\left(z_{0}\right)}{z_{0} g^{\prime}\left(z_{0}\right)}\right\}\right)\right] \\
& =\tan ^{-1} \frac{\alpha \beta k \cos \left\{\operatorname{Arg} \frac{g\left(z_{0}\right)}{z_{0} g^{\prime}\left(z_{0}\right)}\right\}}{1-\alpha \beta k \sin \left\{\operatorname{Arg} \frac{g\left(z_{0}\right)}{z_{0} g^{\prime}\left(z_{0}\right)}\right\}} \\
& \geq \tan ^{-1} \frac{\alpha \beta \cos \left\{\operatorname{Arg} \frac{g\left(z_{0}\right)}{z_{0} g^{\prime}\left(z_{0}\right)}\right\}}{1-\alpha \beta \sin \left\{\operatorname{Arg} \frac{g\left(z_{0}\right)}{z_{0} g^{\prime}\left(z_{0}\right)}\right\}}
\end{aligned}
$$


This contradicts hypothesis (1.11). For the case $\operatorname{Arg} p\left(z_{0}\right)=-\alpha \pi / 2$, applying the same method as the above, we obtain a $k \leq-1$ and so

$$
\operatorname{Arg}\left\{1+\frac{z_{0} p^{\prime}\left(z_{0}\right)}{p\left(z_{0}\right)} \frac{g\left(z_{0}\right)}{z_{0} g^{\prime}\left(z_{0}\right)}\right\} \leq-\tan ^{-1} \frac{\alpha \beta \cos \left\{\operatorname{Arg} \frac{g\left(z_{0}\right)}{z_{0} g^{\prime}\left(z_{0}\right)}\right\}}{1-\alpha \beta \sin \left\{\operatorname{Arg} \frac{g\left(z_{0}\right)}{z_{0} g^{\prime}\left(z_{0}\right)}\right\}}
$$

This contradicts hypothesis (1.11) too and so it completes the proof.

Now, we prove another improvement of Corollary 1.3.

Theorem 1.7 Let $f(z)=z+\sum_{n=2}^{\infty} a_{n} z^{n}$ be in $\mathcal{A}$. If

$$
\left|\operatorname{Arg}\left\{f^{\prime}(z)\right\}\right|<\frac{\pi}{2}\left(\alpha+\frac{2}{\pi} \tan ^{-1} \alpha\right), \quad z \in \mathbb{D},
$$

for some $\alpha, 0<\alpha$, then

$$
\left|\operatorname{Arg}\left\{\frac{f(z)}{z}\right\}\right|<\frac{\alpha \pi}{2}, \quad z \in \mathbb{D} .
$$

Proof Let us write

$$
p(z)=\frac{f(z)}{z}, \quad p(0)=1, \quad z \in \mathbb{D} .
$$

Then $p \in \mathcal{H}$ and it is the form

$$
p(z)=1+\sum_{n=2}^{\infty} a_{n} z^{n-1}
$$

If there exists a point $z_{0},\left|z_{0}\right|<1$ such that

$$
|\operatorname{Arg}\{p(z)\}|<\pi \alpha / 2, \quad \text { in }|z|<\left|z_{0}\right|,
$$

and

$$
\left|\operatorname{Arg}\left\{p\left(z_{0}\right)\right\}\right|=\pi \alpha / 2,
$$

for some $\alpha, 0<\alpha$, then from [4], we have

$$
\frac{z_{0} p^{\prime}\left(z_{0}\right)}{p\left(z_{0}\right)}=i k \alpha
$$

for some $k$, where $k \geq 1$ when $\operatorname{Arg}\left\{p\left(z_{0}\right)\right\}=\alpha \pi / 2$ while $k \leq-1$ when $\operatorname{Arg}\left\{p\left(z_{0}\right)\right\}=$ $-\alpha \pi / 2$. From the equality $f(z)=z p(z)$, we have

$$
f^{\prime}(z)=p(z)+z p^{\prime}(z)=p(z)\left\{1+\frac{z p^{\prime}(z)}{p(z)}\right\},
$$


and so, for the case $\operatorname{Arg}\left\{p\left(z_{0}\right)\right\}=\alpha \pi / 2$, we have

$$
\begin{aligned}
\operatorname{Arg}\left\{f^{\prime}\left(z_{0}\right)\right\} & =\operatorname{Arg}\left\{p\left(z_{0}\right)\left\{1+\frac{z_{0} p^{\prime}\left(z_{0}\right)}{p\left(z_{0}\right)}\right\}\right\} \\
& =\operatorname{Arg}\left\{p\left(z_{0}\right)\right\}+\operatorname{Arg}\left\{1+\frac{z_{0} p^{\prime}\left(z_{0}\right)}{p\left(z_{0}\right)}\right\} \\
& =\frac{\pi \alpha}{2}+\operatorname{Arg}\{1+i \alpha k\} \\
& \geq \frac{\pi \alpha}{2}+\tan ^{-1} \alpha \\
& =\frac{\pi}{2}\left(\alpha+\frac{2}{\pi} \tan ^{-1} \alpha\right) .
\end{aligned}
$$

This contradicts the hypothesis (1.16) and for the case $\operatorname{Arg}\left\{p\left(z_{0}\right)\right\}=-\alpha \pi / 2$, applying the same method as the above, we have

$$
\operatorname{Arg}\left\{f^{\prime}\left(z_{0}\right)\right\} \leq-\frac{\pi}{2}\left(\alpha+\frac{2}{\pi} \tan ^{-1} \alpha\right)
$$

This also contradicts the hypothesis (1.16) and so we obtain that (1.17) holds true.

$$
\begin{aligned}
\operatorname{Arg}\left\{1+\frac{z_{0} p^{\prime}\left(z_{0}\right)}{p\left(z_{0}\right)} \frac{g\left(z_{0}\right)}{z_{0} g^{\prime}\left(z_{0}\right)}\right\} & =\operatorname{Arg}\left\{1+i \alpha k \frac{g\left(z_{0}\right)}{z_{0} g^{\prime}\left(z_{0}\right)}\right\}=\tan ^{-1} \frac{\left(\beta \cos \frac{\pi \gamma}{2}\right) k}{1+\left(\beta \sin \frac{\pi \gamma}{2}\right) k} \\
& \geq \tan ^{-1} \frac{\beta \cos \frac{\pi \gamma}{2}}{1+\beta \sin \frac{\pi \gamma}{2}} .
\end{aligned}
$$

Remark 1 Theorem 1.7 shows that

$$
\left|\operatorname{Arg}\left\{f^{\prime}(z)\right\}\right|<\frac{\pi \beta}{2} \Rightarrow\left|\operatorname{Arg}\left\{\frac{f(z)}{z}\right\}\right|<\frac{\pi}{2}\left(\beta-\frac{2}{\pi} \tan ^{-1} \alpha\right)<\frac{\pi \beta}{2}
$$

in $\mathbb{D}$, where $\beta=\alpha+\frac{2}{\pi} \tan ^{-1} \alpha$, for some $\alpha, 0<\alpha$.

Remark 2 In [6], Pommerenke supposed that $0<\alpha \leq 1$ but in Theorem 1.7 we supposed $0<\alpha$ only.

Theorem 1.8 Let $f(z)=z+\sum_{n=2}^{\infty} a_{n} z^{n}$ be in $\mathcal{K}_{\alpha}$ for some $\alpha, 0<\alpha \leq 1$. Suppose also that

$$
\left|\operatorname{Arg}\left\{f^{\prime}(z)\right\}\right|<\frac{\pi}{2}\left\{\alpha+\frac{2}{\pi} \tan ^{-1} \alpha\right\}, \quad z \in \mathbb{D} .
$$

If

$$
2 \alpha+\frac{2}{\pi} \tan ^{-1} \alpha \leq 1
$$


then $f(z)$ is starlike. If

$$
2 \alpha+\frac{2}{\pi} \tan ^{-1} \alpha>1
$$

then $f(z)$ is starlike in $|z|<\left|z_{0}\right|$, where $\left|z_{0}\right|$ is the smallest positive root of the equation

$$
|z|^{2} \sin \left(\frac{\pi}{2\left(2 \alpha+\frac{2}{\pi} \tan ^{-1} \alpha\right)}\right)-2|z|+\sin \left(\frac{\pi}{2\left(2 \alpha+\frac{2}{\pi} \tan ^{-1} \alpha\right)}\right)=0,
$$

which has the form

$$
\left|z_{0}\right|=\tan \left(\frac{\pi}{4\left(2 \alpha+\frac{2}{\pi} \tan ^{-1} \alpha\right)}\right)<1 .
$$

Proof From Theorem 1.7, we have

$$
\left|\operatorname{Arg} \frac{f(z)}{z}\right|<\frac{\pi \alpha}{2}, \quad z \in \mathbb{D}
$$

Therefore, we have

$$
\begin{aligned}
\left|\operatorname{Arg} \frac{z f^{\prime}(z)}{f(z)}\right| & \leq\left|\operatorname{Arg}\left\{f^{\prime}(z)\right\}\right|+\left|\operatorname{Arg} \frac{f(z)}{z}\right| \\
& <\frac{\pi}{2}\left(2 \alpha+\frac{2}{\pi} \tan ^{-1} \alpha\right) .
\end{aligned}
$$

If $\alpha$ satisfies (1.19), then (1.23) follows that $f(z)$ is a starlike function. If $\alpha$ satisfies (1.20), then applying Lemma 1.2, we have

$$
\left|\operatorname{Arg} \frac{z f^{\prime}(z)}{f(z)}\right|<\left(2 \alpha+\frac{2}{\pi} \tan ^{-1} \alpha\right) \sin ^{-1} \frac{2|z|}{1+|z|^{2}}<\frac{\pi}{2}
$$

for some small $|z|$. It follows that $f(z)$ is starlike in $|z|<\left|z_{0}\right|$, where $\left|z_{0}\right|$ is positive root of the equation (1.21). Simple calculation shows that

$$
\left|z_{0}\right|=\frac{1-\sqrt{1-c^{2}}}{c}, \quad \text { where, } \quad c=\sin \left(\frac{\pi}{2\left(2 \alpha+\frac{2}{\pi} \tan ^{-1} \alpha\right)}\right),
$$

so we obtain (1.22).

Theorem 1.9 Let $f(z)=z+\sum_{n=2}^{\infty} a_{n} z^{n}$ be in $\mathcal{K}_{\alpha}$ for $\alpha, 0<\alpha \leq 1$. Then $f(z)$ is starlike in

$$
|z|<\tan \frac{\pi}{4(1+2 \alpha)}<1 .
$$

Proof From Lemma 1.1 and hypothesis of the Theorem, we have

$$
\left|\operatorname{Arg} \frac{z f^{\prime}(z)}{f(z)}\right|<\frac{\pi}{2}(1+2 \alpha), \quad z \in \mathbb{D}
$$


and hence from Lemma 1.2, we have

$$
\left|\operatorname{Arg} \frac{z f^{\prime}(z)}{f(z)}\right|<(1+2 \alpha) \sin ^{-1} \frac{2|z|}{1+|z|^{2}}, \quad z \in \mathbb{D} .
$$

Putting

$$
(1+2 \alpha) \sin ^{-1} \frac{2|z|}{1+|z|^{2}}<\frac{\pi}{2}
$$

we have

$$
\left|\operatorname{Arg} \frac{z f^{\prime}(z)}{f(z)}\right|<\frac{\pi}{2}, \quad z \in \mathbb{D}
$$

for $|z|$ as in (1.24).

Remark 3 The radius of starlikeness in the class $\mathcal{K}_{\alpha}$ may be equal or larger than the above value. This is an open question.

We say that $f(z) \in \mathcal{K}_{\beta}^{\alpha}$ whenever $f(z) \in \mathcal{A}$ and there exist a real $0<\alpha \leq 2$ and $0<\beta \leq 1$ and a function $g(z) \in \mathcal{K}$ such that

$$
\left|\operatorname{Arg} \frac{f^{\prime}(z)}{g^{\prime}(z)}\right|<\frac{\alpha \pi}{2}, \quad z \in \mathbb{D}
$$

and

$$
\left|\operatorname{Arg} \frac{z g^{\prime}(z)}{g(z)}\right|<\frac{\beta \pi}{2}
$$

For $\beta \in(0,1]$ the function

$$
g(z)=z \exp \int_{0}^{z} \frac{(1+t)^{\beta}-1}{t} \mathrm{~d} t, \quad z \in \mathbb{D}
$$

is in $\mathcal{K}$ because

$$
\mathfrak{R e}\left\{1+\frac{z g^{\prime \prime}(z)}{g^{\prime}(z)}\right\}=\mathfrak{R e}\left\{(1+z)^{\beta}+\frac{\beta}{1+z}\right\}>\frac{\beta}{2}, \quad z \in \mathbb{D},
$$

and

$$
\left|\operatorname{Arg} \frac{z g^{\prime}(z)}{g(z)}\right|=\left|\operatorname{Arg}\left\{(1+z)^{\beta}\right\}\right|<\frac{\beta \pi}{2} .
$$

Therefore, a function $f(z)$ such that $f(z) \equiv g(z)$ is in the class $\mathcal{K}_{\beta}^{\alpha}$ for all $0<\alpha \leq 2$. For this class $\mathcal{K}_{\beta}^{\alpha}$ holds the following theorem under some restriction on $\alpha$ and $\beta$.

Theorem 1.10 Let $f(z)=z+\sum_{n=2}^{\infty} a_{n} z^{n}$ be in $\mathcal{K}_{\beta}^{\alpha}$ for some $\alpha>0$ and $0<\beta \leq 1$. If $2 \alpha+\beta \leq 1$, then $f(z)$ is starlike in $\mathbb{D}$, if $2 \alpha+\beta>1$, then $f(z)$ is starlike in

$$
|z|<\tan \frac{\pi}{4(\beta+2 \alpha)}<1
$$


Proof From (1.25), (1.26) and from (1.5), we have

$$
\begin{aligned}
\left|\operatorname{Arg} \frac{z f^{\prime}(z)}{f(z)}\right| & =\left|\operatorname{Arg}\left\{\frac{f^{\prime}(z)}{g^{\prime}(z)} \frac{z g^{\prime}(z)}{g(z)} \frac{g(z)}{f(z)}\right\}\right| \\
& \leq\left|\operatorname{Arg}\left\{\frac{f^{\prime}(z)}{g^{\prime}(z)}\right\}\right|+\left|\operatorname{Arg}\left\{\frac{z g^{\prime}(z)}{g(z)}\right\}\right|+\left|\operatorname{Arg}\left\{\frac{g(z)}{f(z)}\right\}\right| \\
& \leq \frac{\alpha \pi}{2}+\frac{\beta \pi}{2}+\frac{\alpha \pi}{2} \\
& =\frac{\pi}{2}(2 \alpha+\beta), \quad z \in \mathbb{D} .
\end{aligned}
$$

If $2 \alpha+\beta \leq 1$, then $f(z)$ is starlike in $\mathbb{D}$ because in this case (1.28) is lees than $\pi / 2$. For the case $2 \alpha+\beta>1$ we can apply the same method as in the proof of Theorem 1.9 to obtain (1.27).

Theorem 1.11 Let $f(z), g(z)$ be in $\mathcal{A}$. Suppose that

$$
\left|\operatorname{Arg} \frac{f^{\prime}(z)}{g^{\prime}(z)}\right|<\frac{\pi}{2}(\alpha-\beta-\gamma), \quad z \in \mathbb{D}
$$

and

$$
\left|\operatorname{Arg} \frac{z f^{\prime}(z)}{f(z)}\right|<\frac{\gamma \pi}{2}, \quad\left|\operatorname{Arg} \frac{z g^{\prime}(z)}{g(z)}\right|<\frac{\beta \pi}{2}, \quad z \in \mathbb{D},
$$

where $0<\alpha-\beta-\gamma, 0<\beta$ and $0<\gamma$. Then we have

$$
\left|\operatorname{Arg} \frac{f(z)}{g(z)}\right|<\frac{\alpha \pi}{2}, \quad z \in \mathbb{D} \text {. }
$$

Proof Putting $p(z)=f(z) / g(z), z \in \mathbb{D}$, gives $p(0)=1$ and $f(z)=p(z) g(z)$. It follows that

$$
\frac{f^{\prime}(z)}{g^{\prime}(z)}=p(z)\left(1+\frac{z p^{\prime}(z)}{p(z)} \frac{g(z)}{z g^{\prime}(z)}\right)=p(z) \frac{z f^{\prime}(z)}{f(z)} \frac{g(z)}{z g^{\prime}(z)} .
$$

If there exists a point $z_{0} \in \mathbb{D}$ such that

$$
|\operatorname{Arg}\{p(z)\}|<\frac{\alpha \pi}{2} \text { for }|z|<\left|z_{0}\right|
$$

and

$$
\left|\operatorname{Arg}\left\{p\left(z_{0}\right)\right\}\right|=\frac{\alpha \pi}{2},
$$

then we have 


$$
\begin{aligned}
\left|\operatorname{Arg} \frac{f^{\prime}\left(z_{0}\right)}{g^{\prime}\left(z_{0}\right)}\right| & \geq\left|\operatorname{Arg}\left\{p\left(z_{0}\right)\right\}\right|-\left|\operatorname{Arg} \frac{z_{0} f^{\prime}\left(z_{0}\right)}{f\left(z_{0}\right)}\right|-\left|\operatorname{Arg} \frac{z_{0} g^{\prime}\left(z_{0}\right)}{g\left(z_{0}\right)}\right| \\
& >\frac{\alpha \pi}{2}-\frac{\beta \pi}{2}-\frac{\gamma \pi}{2} .
\end{aligned}
$$

This contradicts the hypothesis, and it completes the proof.

Some related condition for starlikeness can be found from [9].

Acknowledgements The authors express their sincerest thanks to the referee for the invaluable suggestions.

\section{Compliance with ethical standards}

Conflict of interest The authors declare that they have no conflict interest.

Open Access This article is distributed under the terms of the Creative Commons Attribution 4.0 International License (http://creativecommons.org/licenses/by/4.0/), which permits unrestricted use, distribution, and reproduction in any medium, provided you give appropriate credit to the original author(s) and the source, provide a link to the Creative Commons license, and indicate if changes were made.

\section{References}

1. Brannan, D.A., Kirwan, W.E.: On some classes of bounded univalent functions. J. Lond. Math. Soc. 1(2), 431-443 (1969)

2. MacGregor, T.H.: A subordination for convex function of order $\alpha$. J. Lond. Math. Soc. 2(9), 530-536 (1975)

3. Nunokawa, M.: On properties of non-Carathèodory functions. Proc. Jpn. Acad. Ser. A 68(6), 152-153 (1992)

4. Nunokawa, M.: On the order of strongly starlikeness of strongly convex functions. Proc. Jpn. Acad. Ser. A 69(7), 234-237 (1993)

5. Nunokawa, M., Sokół, J.: On an extension of Sakaguchi’s result. J. Math. Inequal. 9(3), 683-697 (2015)

6. Pommerenke, Ch.: On close to-convex analytic functions. Trans. Am. Math. Soc. 114(1), 176-186 (1965)

7. Ponnusamy, S., Karunakaran, V.: Differential subordination and conformal mappings. Complex Var. Theory Appl. 11(2), 79-86 (1989)

8. Sakaguchi, K.: On a certain univalent mapping. J. Math. Soc. Jpn. 11(1), 72-75 (1959)

9. Sokół, J., Nunokawa, M.: On some sufficient condition for starlikeness. J. Inequal. Appl. 2013, 282 (2013)

10. Stankiewicz, J.: Quelques problèmes extrémaux dans les classes des fonctions $\alpha$-angulairement étoilées. Ann. Univ. Mariae Curie-Skłodowska Sect. A 20(1966), 59-75 (1971)

11. Study, E.: Konforme Abbildung Einfachzusammenhangender Bereiche. B. C. Teubner, Leipzig (1913)

Publisher's Note Springer Nature remains neutral with regard to jurisdictional claims in published maps and institutional affiliations. 